\title{
Alterações no sistema nervoso central e suas manifestações neuropsiquiátricas em pacientes pós COVID-19
}

\author{
Changes in the central nervous system and its neuropsychiatric manifestations in patients \\ after COVID-19
}

\section{Cambios en el sistema nervioso central y sus manifestaciones neuropsiquiátricas en pacientes después de COVID-19}

Talita Miranda de Amorim ${ }^{1 *}$, Anne Caroline Rogienfisz Mendes ${ }^{2}$, Carolina de Souza Fernandes Correa $^{2}$, Fernando Lonardelli Saraiva Ramalho ${ }^{3}$, Igor Andrade Silveira ${ }^{1}$, Mariana Medeiros de Souza $^{1}$, Thainara de Oliveira Magalhães ${ }^{1}$, Sofia Soares Rosendo ${ }^{1}$, Samara Darissi Cruz ${ }^{1}$, Manoel Victor Vasconcelos Miranda Guzella ${ }^{4}$.

\section{RESUMO}

Objetivo: Discutir as alterações neurológicas e psiquiátricas em pacientes pós COVID-19, a partir de uma revisão narrativa sobre o tema, relacionando os acometimentos causados pelo vírus no Sistema Nervoso Central (SNC) e suas manifestações neuropsiquiátricas. Revisão bibliográfica: A Covid-19 trata-se de uma infecção respiratória aguda grave de alta transmissibilidade causada por uma nova espécie de coronavírus, denominada SARS-CoV-2 que tem o pulmão como órgão de predileção. Além disso, os vírus também podem penetrar no SNC e causar diversas patologias neurológicas e manifestações neuropsiquiátricas como, por exemplo, acidente vascular, convulsões, encefalite, ansiedade, depressão. É evidente ainda o desenvolvimento de transtornos mentais que estão relacionados, principalmente, ao isolamento e distanciamento social ocasionados pela pandemia. Considerações finais: É de extrema importância o acompanhamento neuropsiquiátrico durante e após a infecção pelo COVID-19, a fim de realizar uma intervenção precoce e dessa forma, poder melhorar o prognóstico dos indivíduos acometidos pelo vírus. Entretanto, essas alterações tratam-se de um tema recente no escopo da medicina e ainda há muito o que ser estudado pelos pesquisadores.

Palavras-chave: Coronavirus, Sistema nervoso, Saúde mental.

\begin{abstract}
Objective: Discuss neurological and psychiatric disorders in post-COVID-19 patients, based on a narrative review of the topic, relating the affections caused by the virus in the Central Nervous System (CNS) and its neuropsychiatric manifestations. Bibliographic review: Covid-19 is a severe, highly transmissible, acute respiratory infection caused by a new species of coronavirus, called SARS-CoV-2, which has the lung as a preferred organ. In addition, viruses can also penetrate the CNS and cause various neurological pathologies and neuropsychiatric manifestations such as stroke, seizures, encephalitis, anxiety, depression. It is also evident the development of mental disorders that are mainly related to the isolation and social distance caused by the pandemic. Final considerations: Neuropsychiatric follow-up during and after infection by COVID-19 is extremely important, in order to perform an early intervention and thus be able to improve the prognosis of individuals affected by the virus. However, these changes are a recent topic in the scope of medicine and there is still much to be studied by researchers.
\end{abstract}

Key words: Coronavirus, Nervous system, Mental health.

\footnotetext{
1 Faculdade Vértice (UNIVÉRTIX), Matipó - MG. *E-mail: talitaamorim0@gmail.com

2 Universidade Fundação Oswaldo Aranha (UniFOA), Volta Redonda - RJ.

${ }^{3}$ Faculdade Ciências Médicas de Minas Gerais (FCCMG), Belo Horizonte - MG.

${ }^{4}$ Centro Universitário de Caratinga (UNEC), Caratinga - MG.
} 


\section{RESUMEN}

Objetivo: Discutir los trastornos neurológicos y psiquiátricos en pacientes post-COVID-19, a partir de una revisión narrativa sobre el tema, relacionando las afecciones causadas por el virus en el Sistema Nervioso Central (SNC) y sus manifestaciones neuropsiquiátricas. Revisión bibliográfica: Covid-19 es una infección respiratoria aguda grave, altamente transmisible, causada por una nueva especie de coronavirus, llamado SARS-CoV-2, que tiene al pulmón como órgano preferido. Además, los virus también pueden penetrar en el SNC y provocar diversas patologías neurológicas y manifestaciones neuropsiquiátricas como apoplejía, convulsiones, encefalitis, ansiedad, depresión. También es evidente el desarrollo de trastornos mentales que se relacionan principalmente con el aislamiento y la distancia social provocada por la pandemia. Consideraciones finales: El seguimiento neuropsiquiátrico durante y después de la infección por COVID-19 es de suma importancia para realizar una intervención temprana y así poder mejorar el pronóstico de las personas afectadas por el virus. Sin embargo, estos cambios son un tema reciente en el ámbito de la medicina y aún queda mucho por estudiar por parte de los investigadores.

Palabras clave: Coronavirus, Sistema nervioso, Salud mental.

\section{INTRODUÇÃO}

A COVID-19 é uma infecção respiratória aguda, potencialmente grave, com alta transmissibilidade e distribuição global. O órgão mais afetado por essa doença é o pulmão, causando uma pneumonite com perda da capacidade de fazer trocas gasosas, em especial ao inspirar. Até o momento, foi evidenciado a existência de quadros com variabilidade de agravamento, com casos assintomáticos, moderados, graves e críticos. Essa doença pode, ainda, apresentar graves complicações como falência respiratória, sepse, choque séptico, tromboembolismo, falência múltipla dos órgãos, lesão hepática ou cardíaca aguda e óbito (TESINI BL, 2020).

Até o mês de junho de 2021, foram confirmados mais de 182 milhões casos de COVID-19 no mundo. Em relação ao Brasil, o Ministério da Saúde recebeu a primeira notificação de um caso confirmado no dia 26 de fevereiro de 2020 e, até o mesmo período, foram confirmados mais de 18 milhões de casos e cerca de 500 mil óbitos por COVID-19 no país (BRASIL, 2021). A transmissão do novo Coronavírus ocorre de pessoa para pessoa principalmente por meio de gotículas respiratórias que são liberadas quando o indivíduo infectado tosse ou espirra, e também por contato direto, semelhante ao vírus da influenza humana, Síndrome Respiratória Aguda Grave (SARS-CoV) e a Síndrome Respiratória do Oriente Médio (MERS-CoV) (GUAN WJ, et al., 2020).

À medida que a pandemia da SARS-CoV-2 progride, evidências mostram que este vírus pode cursar com neuroinvasão e afetar o sistema nervoso central de várias maneiras (DESFORGES M, et al., 2019). Assim, estudos têm demonstrado que as manifestações neurológicas durante a infecção podem ser causadas diretamente pelo vírus no SNC por desregulação da rede de citocinas, transmigração de células imunológicas periféricas e resposta autoimune pós-infecciosa. Além disso, após obter acesso ao SNC, o vírus pode causar infiltração de células imunes do hospedeiro e ativar vias inflamatórias e, assim, ativar vias trombóticas que contribuem para o dano tecidual e causam microangiopatia (ELLUL MA, et al., 2020; NALLEBALLE K, et al., 2020; VARGA Z, et al., 2020).

Dessa forma, é notório que alguns pacientes recuperados apresentam sequelas relacionadas diretamente à questões neurológicas como: acidente vascular cerebral isquêmico, hemorragia intracerebral, hemorragia subaracnóidea, trombose de seio venoso, vasculite cerebral, encefalopatia, encefalite, convulsões, síndromes neuropsiquiátricas (psicose, síndrome neurocognitiva semelhante à demência, alterações de personalidade, catatonia, ansiedade, depressão, síndrome de fadiga crônica e estresse pós traumático), síndrome de Guillain Barré, síndrome de Miller Fisher, neuritebraquial, miastenia gravis, neuropatia periférica, miopatia, miosite e neuromiopatia do doente crítico (SULTANA S e ANANTHAPUR V, 2020).

Além disso, manifestações neuropsiquiátricas pós infecção têm sido descritas, em virtude, principalmente, das medidas de restrição e isolamento social necessárias à contenção da transmissão viral. Dentre elas, as 
mais recorrentes são o estresse pós-traumático, depressão, solidão, raiva, ansiedade, sintomas obsessivoscompulsivos, transtornos do humor, dor de cabeça e transtornos do sono (NALLEBALLE K, et al., 2020).

Desse modo, o objetivo deste artigo é discutir as alterações neurológicas e psiquiátricas em pacientes pós COVID-19, a partir a partir de uma revisão narrativa sobre o tema.

\section{REVISÃO BIBLIOGRÁFICA}

De dezembro de 2019 a janeiro de 2020, a Organização Mundial da Saúde (OMS) foi notificada pela Comissão Nacional de Saúde da China sobre a existência de um surto de pneumonia com etiologia desconhecida. Posteriormente, tal condição foi identificada como um novo tipo de coronavírus e denominada como Síndrome da Respiratória Aguda Grave 2 (SARS-CoV-2). A partir desse momento, em 11 de março de 2020, a OMS caracterizou o episódio como pandemia e elaborou medidas preventivas para conter o avanço da doença (OMS, 2020).

O coronavírus-19 (COVID-19) apresenta a patogênese principal pelo epitélio pulmonar. Essa característica faz com que a doença se manifeste comumente como uma afecção respiratória. Logo, os sintomas típicos incluem tosse, falta de ar e fadiga (ADIL MT, et al., 2020). O coronavírus afeta a regulação da perfusão pulmonar, ocasionando danos alveolares, levando a angiogênese e a formação de trombos. Com isso, devido às alterações na respiração e a hipoxemia, torna-se necessária a utilização da ventilação mecânica em casos mais graves da doença (HABASHI NM, et al., 2021).

Apesar do COVID-19 ter ganhado destaque como patologia predominantemente do sistema respiratório, também é possível enfatizar seus impactos neurológicos e psiquiátricos. Estudos mostram que o vírus é neuroinvasivo e neurotóxico, ou seja, é capaz de se apresentar com grande carga viral no sistema nervoso, visto que se difunde por meio da barreira hematoencefálica, utilizando-se do transporte axonal retrógrado. Essa situação é capaz de ocasionar lesões neuronais consideráveis, que podem evoluir para acidentes vasculares encefálicos (AVE), comprometimento da visão, epilepsia, encefalite, aneurisma, síndrome de Guillain-Barré, entre outras doenças (SULTANA S e ANANTHAPUR V, 2020).

Alguns pesquisadores discorrem sobre a possibilidade do SARS-CoV-2 invadir o cérebro através do nervo olfatório, espalhando-se para o tronco encefálico e outras áreas do sistema nervoso, bem como para o líquido cefalorraquidiano, sendo uma provável justificativa da hiposmia ser um dos primeiros sintomas neurológicos observados no COVID-19. Eles defendem, ainda, que as dificuldades na respiração espontânea, característica dos casos mais graves, possa ser em decorrência da disseminação da infecção para o tronco encefálico. O impacto causado pelo vírus no sistema nervoso pode ser muito maior e mais devastador do que o impacto nos pulmões, pois pacientes que não apresentaram complicações iniciais durante a infecção passaram a ter, posteriormente, manifestações de desorientação, perda de memória e quadros neurológicos críticos ou irreversíveis como ablepsia, podendo até mesmo evoluir a óbito (MUKAETOVA-LADINSKA EB e KRONENBERG G, 2020).

Helms J, et al. (2020) confirmam que as primeiras descrições da epidemia na China já relatavam a neurotoxicidade do COVID-19, com quase metade dos pacientes gravemente enfermos apresentando alguns sintomas neurológicos, e $81 \%$ daqueles na terapia intensiva apresentando essa sintomatologia. Esses dados corroboram a prevalência relatada de afecções neurológicas em um estudo conduzido em 214 pacientes, dos quais $36,4 \%$ apresentavam sintomas neurológicos. Szcześniak D, et al. (2021) observaram que, já nos primórdios da pandemia, havia relatos mostrando que o SARS-CoV-2 pode danificar o SNC, causando encefalite viral, encefalopatia hipóxica, meningoencefalite, encefalopatia tóxica infecciosa e doença cerebrovascular aguda.

Os sintomas neurológicos na fase aguda, geralmente são leves e de natureza transitória, podendo incluir cefaléia, náuseas, vômitos, confusão, hiposmia, hipogeusia e sintomas musculoesqueléticos. O AVE foi descrito mesmo em formas mais leves de COVID-19, sugerindo que esses pacientes estão em maior risco de formação de trombos, o que pode estar associado, em grande parte, a danos causados pelo vírus ao endotélio do vaso. A encefalite viral também pode representar os primeiros sinais de infecção por COVID-19, indicando 
que a SARS-CoV-2 pode afetar o tecido cerebral central, tanto como resultado de uma invasão direta do sistema nervoso quanto de sua resposta imunológica (MUKAETOVA-LADINSKA EB e KRONENBERG G, 2020).

No momento, não é possível prever as consequências neurológicas de longo prazo do COVID-19 com base na sintomatologia que os pacientes apresentam durante a fase aguda. No entanto, é possível que pacientes mais gravemente afetados pela infecção viral tenham um risco aumentado de comprometimento cognitivo, que pode estar ligado à neurodegeneração cortical ou subcortical, bem como a mecanismos vasculares (SZCZEŚNIAK D, et al., 2021).

Outro dado relevante encontrado foi que as contagens de linfócitos eram menores e com níveis de dímeroD mais elevados em pacientes com sintomas do SNC, o que pode explicar porque os pacientes com manifestação grave desenvolvem doença cerebrovascular. No entanto, a incidência desses sintomas foi paralela à gravidade da doença, com até $88 \%$ dos pacientes gravemente enfermos exibindo clínica envolvendo o sistema nervoso central e periférico, bem como os músculos esqueléticos, especialmente doença cerebrovascular aguda, distúrbios de consciência e lesão muscular (MAO L, et al., 2020).

Um outro estudo, desta vez de análise retrospectiva, realizado em uma rede hospitalar de Chicago nos Estados Unidos, concluiu que a encefalopatia foi a manifestação neurológica que trouxe consigo as maiores complicações clínicas nos pacientes com COVID-19 avaliados. A pesquisa observou, ainda, que o acometimento encefálico foi mais frequente em pacientes idosos, tendo uma taxa de mortalidade de $21,7 \%$ ao longo de 30 dias (LIOTTA EM, et al., 2020).

É possível verificar essas alterações neurológicas a partir de exames complementares. A ressonância magnética do cérebro, por exemplo, mostra aumento dos espaços subaracnóideos e anormalidades de perfusão. O eletroencefalograma é capaz de revelar anormalidades inespecíficas ou atividade difusa lenta. $O$ exame do líquido cefalorraquidiano revela distúrbios inflamatórios, demonstrando a presença de bandas oligoclonais e níveis elevados de proteínas e IgG. Evidências atuais sugerem, ainda, que a presença de SARS-CoV-2 no líquor pode depender da gravidade da doença e do grau de tropismo do tecido nervoso do vírus (SZCZESNIAK D, et al., 2021).

É bem estabelecido que os pacientes com infecção por COVID-19 com história prévia de doença cerebrovascular têm maior risco de manifestar quadros graves do que aqueles sem essas doenças, incluindo a necessidade de internação em terapia intensiva e ventilação mecânica, bem como maior taxa de mortalidade. Embora a evidência dos sintomas neurológicos do COVID-19 pareça ter se estabilizado e esteja junto com os sintomas neurológicos virais já conhecidos, as sequelas de saúde mental permanecem indefinidas (TARSITANI L, et al., 2021).

Taquet M, et al. (2021) realizaram um estudo de coorte que demonstrou a associação entre o coronavírus e doenças psiquiátricas e neurológicas. A metodologia dessa pesquisa se baseou na análise do tempo de diagnóstico do SARS-CoV-2 até o surgimento dos sintomas neurológicos e psiquiátricos. Os autores observaram que a incidência aproximada dessas manifestações após seis meses da doença foi de 33,6\%. Ainda, em relação a análise individual, houve ocorrência de 0,56\% para hemorragia intracraniana, 2,1\% para o acidente vascular cerebral isquêmico, $0,1 \%$ para parkinsonismo, $0,67 \%$ para demência, $17,4 \%$ para transtorno de ansiedade e 1,4\% para transtorno psicótico. Szcześniak D, et al. (2021) analisaram um estudo com 40.469 pacientes diagnosticados com COVID-19, em que $22,5 \%$ deles expressaram sintomas neuropsiquiátricos, incluindo cefaleia $(3,7 \%)$, insônia $(3,4 \%)$, encefalopatia $(2,3 \%)$, doença cerebrovascular $(1 \%)$, depressão $(3,8 \%)$ e ideação suicida $(0,2 \%)$.

Outro importante achado na literatura diz respeito à elevada ocorrência de transtornos psiquiátricos em indivíduos que contraíram COVID-19 (TARSITANI L, et al., 2021). Butler M, et al. (2020) atestaram que sintomas neuropsiquiátricos, particularmente delirium, já eram comuns em surtos anteriores de outros coronavírus de doença respiratória aguda grave (SARS) e síndrome respiratória do Oriente Médio (MERS) e que, até agora, o COVID-19 parece seguir um padrão semelhante.

Nos casos atuais, pacientes têm manifestado delirium como sendo a síndrome neuropsiquiátrica aguda mais comum e podendo ser a única ou o primeiro sintoma clínico, característico de apresentação de COVID- 
10 em idosos, especialmente naqueles com demência. O delirium está, ainda, associado a piores resultados sendo especialmente prevalente entre os pacientes que requerem cuidados intensivos, onde anormalidades cognitivas e comportamentais foram relatadas em um terço dos pacientes após a alta hospitalar (BUTLER M, et al., 2020).

Helms J, et al. (2020) foram responsáveis por um estudo francês que relatou a incidência de delirium em casos de COVID-19 variando de 9 a 14,8\%. Na UTI, 69,3\% dos doentes exibiram delirium hiperativo, apesar das altas taxas de infusão de tratamentos sedativos e neurolépticos. Um estudo mais recente, usando o Método de Avaliação de Confusão para a UTI (Confusion Assessment Method for the ICU), relatou que o delirium estava presente em $75,9 \%$ dos pacientes com COVID-19 internados. Assim, é possível especular que 32 a $44 \%$ dos pacientes com COVID-19 intensamente ventilados mecanicamente podem ter tido delirium não reconhecido ou registrado, o que faz com que os dados relatados provavelmente subestimem a extensão do delirium no COVID-19, especialmente aqueles com delirium hipoativo e subsindrômico, não sendo diagnosticadas (YANG X, et al., 2020; ZHAO S, et al., 2020).

Especula-se que a psicose ligada a COVID-19 possa surgir como uma resposta direta do cérebro à hipercitocinemia e resposta imune ou ser uma resposta secundária à medicação, sendo que a incidência de psicose entre pessoas com infecção viral varia entre 0,9 e $4 \%$. Um dos relatórios de vigilância do Reino Unido descreveu o estado mental alterado em 31\% dos 139 pacientes com SARS-CoV-2 confirmados, com 59\% dos casos apresentando sintomas neuropsiquiátricos. Embora o estudo não inclua fatores iatrogênicos que possam ter contribuído para o estado mental alterado, ou seja, medicações como corticosteroide, sedativos e antipsicóticos, o início precoce da psicose e a gravidade da infecção viral são sugestivos de fatores precipitantes autoimunes levando ao estado mental alterado (VARATHARAJ A, et al., 2020).

Outro ponto relevante observado diz respeito aos impactos que a pandemia e as medidas associadas a ela geraram na saúde mental da população de forma geral. Um surto repentino de uma doença sempre representa uma ameaça à saúde mental das pessoas afetadas e de seus contatos próximos. Pacientes confirmados, pacientes suspeitos, profissionais de saúde e aqueles com contato próximo aos pacientes podem ter uma prevalência maior de ansiedade, depressão, raiva e outros problemas psicológicos associados (AHMED MZ, et al., 2020).

Além disso, desde o início da pandemia, foram impostas ações e normas para controle da disseminação viral, visando reduzir o contato interpessoal nos principais locais de elevado fluxo de pessoas, como nos espaços de convívio comunitário, comércios, empresas, escolas, transportes públicos, eventos esportivos, culturais. $O$ uso de máscaras e os cuidados de higiene também foram medidas que mudaram o cotidiano de muitas pessoas e essa nova realidade proporcionou a descontinuação das relações sociais (CASAGRANDE M, et al., 2020).

Nessa situação, o medo, a tristeza, a ansiedade e a preocupação são reações consideradas comuns e compreensíveis que podem se manifestar em todas as pessoas. Entretanto, em alguns casos, estas reações podem se prolongar e se tornar mais agravantes ou incapacitantes, levando a um aumento dos transtornos psíquicos na população (BROOKS SK, et al., 2020). É válido ressaltar, ainda, a ocorrência de transtornos de adaptação, que se caracteriza pela tentativa do organismo em manter-se em homeostase frente a situações adversas. Essa situação também pode trazer consequências graves tanto à saúde física quanto à mental (LIPP MEN e LIPP LMN, 2020).

Uma pesquisa realizada pelo Ministério da Saúde sobre a saúde mental da população brasileira observou que $74 \%$ dos entrevistados relataram ansiedade, $26,8 \%$ relataram depressão na sua forma moderada e $12,3 \%$ relataram depressão na sua forma grave durante a pandemia da COVID-19 (BRASIL, 2020). Outros estudos têm encontrado resultados semelhantes, com aumento expressivo e preocupante dos problemas de saúde mental durante este período e indicativos de que, ao longo do tempo, os sintomas psicológicos tendem a ser intensificados e transformados em problemas neurológicos e neuromusculares (BARROS MA, et al., 2020; AHMED MZ, et al., 2020).

Mediante a fisiopatologia da doença, as consequências psicológicas pós COVID-19 raramente requerem um tratamento farmacológico, já as consequências neuropsiquiátricas requerem este tratamento mais preciso. 
As alterações neurológicas devem ser tratadas com acompanhamento médico e indica-se a realização de tomografia e ressonância torácica e encefálica para que se avalie internamente a situação dos órgãos. $O$ tronco encefálico pode ser a parte do cérebro mais afetada, por isso deve-se observar atentamente os sintomas associados (ROGERS JP, et al., 2021).

Em relação à saúde mental, intervenções através de cronogramas de atividades podem ser implementadas para manter a população ativa através de exercícios, mantendo um equilíbrio mental e físico. A Comissão Nacional de Saúde da China enfatiza que os pacientes da COVID-19 necessitam de cuidados da saúde mental, não só destes, como também de seus familiares, médicos e enfermeiros que prestam cuidado a todos os pacientes contaminados (MUKAETOVA-LADINSKA EB, 2020; KRONENBERG G, 2020).

Por fim, ao se analisar as perspectivas futuras relacionadas aos impactos neuropsiquiátricos do SARSCoV-2, é perceptível a necessidade do acompanhamento dos pacientes após a doença de forma a favorecer o tratamento precoce e o melhor prognóstico das implicações neurológicas e psiquiátricas. Entretanto, reforçase a importância do desenvolvimento de mais estudos neste tema, visto que as pesquisas existentes ainda são recentes (TAQUET M, et al., 2021; SULTANA S e ANANTHAPUR V, 2020).

\section{CONSIDERAÇÕES FINAIS}

A partir da presente revisão, observou-se que, apesar da predileção do SARS-CoV-2 pelos pulmões, sabese que inúmeras são alterações neurológicas e psiquiátricas em pacientes acometidos. Tem-se observado também repercussões na saúde mental da população, em decorrência do isolamento e distanciamento social ocasionados pela pandemia. Diante desse difícil momento, é de suma importância compreender melhor a COVID-19 e suas complicações, particularmente as neurológicas, no intuito de obter um maior detalhamento de informações para os bancos de dados, melhores diagnósticos e possibilidades terapêuticas para os pacientes. Além disso, faz-se extremamente necessário o acompanhamento neuropsiquiátrico por todo processo de desenvolvimento da doença e após seu desfecho, a fim de potencializar um melhor prognóstico e tratamento individual e coletivo.

\section{REFERÊNCIAS}

1. ADIL MT, et al. SARS-CoV-2 and the pandemic of COVID-19. Postgraduate Medical Journal, 2021; 97(1144):110116.

2. AHMED MZ, et al. Epidemic of COVID-19 in China and associated Psychological Problems. Asian Journal of Psychiatry,2020;51: 1-7.

3. BARROS MA, et. al. Relato de tristeza/depressão, nervosismo/ansiedade e problemas de sono na população adulta brasileira durante a pandemia de COVID-19. Revista Epidemiologia e Serviços de Saúde, 2020; 29(4):e2020427.

4. BRASIL. Boletim epidemiológico especial. Doença pelo coronavírus covid-19. 2021. Disponível em: https://www.gov.br/saude/pt-br/media/pdf/2021/marco/05/boletim_epidemiologico_covid_52_final2.pdf. Acessado em: 24 de abril de 2021

5. BRASIL. Ministério da Saúde - Segunda etapa da pesquisa inédita realizada pelo Ministério da Saúde abordou procura por atendimento profissional e consumo de álcool e drogas. 2020. Disponível em: https://aps.saude.gov.br/noticia/10658. Acessado em: 20 de maio de 2021.

6. BUTLER M, et al. Neuropsychiatric complications of covid-19: From acute delirium to long term fatigue, covid-19 has serious neuropsychiatric effects. British Medical Journal, 2020; 371: 3871.

7. BROOKS SK, et al. The psychological impact of quarantine and how to reduce it: rapid review of the evidence. The Lancet, 2020; 395: 912-920.

8. CASAGRANDE M, et al. The enemy who sealed the world: effects quarantine due to the COVID-19 on sleep quality, anxiety, and psychological distress in the Italian population. Sleep Medicine, 2020; 75: 12-20.

9. DESFORGES M, et al. Human coronaviruses and other respiratory viruses: underestimated opportunistic pathogens of the central nervous system? Viruses, 2019;12(1):14.

10. ELLUL MA, et al. Neurological associations of COVID-19. Lancet Neurology, 2020;19:767-83.

11. GUAN WJ, et al. Clinical Characteristics of Coronavirus Disease 2019 in China. The New England Journal of Medicine, 2020; 382: 1708-1720.

12. HABASHI NM, et. al. Functional pathophysiology of SARS-CoV-2-induced acute lung injury and clinical implications. Journal of applied physiology, 2021 130(3), 877-891.

13. HELMS J, et al. Neurologic features in severe SARS-CoV-2 infection. The New England Journal of Medicine, 2020 ; 382:2268-2270. 
14. LIOTTA EM, et al. Frequent neurologic manifestations and encephalopathy-associated morbidity in Covid-19 patients. Annals of Clinical and Translational Neurology, 2020; 7(11): 2221-2230.

15. LIPP MEN, LIPP LMN. Stress e transtornos mentais durante a pandemia da COVID-19 no Brasil. Revista da Academia Paulista de Psicologia, 2020; 40(99),180-191.

16. MAO L, et al. Neurologic Manifestations of Hospitalized Patients With Coronavirus Disease 2019 in Wuhan, China. JAMA Neurology. 2020;77(6):683-690.

17. MUKAETOVA-LADINSKA EB, KRONENBERG G. Psychological and neuropsychiatric implications of COVID-19. European Archives of Psychiatry and Clinical Neuroscience, 2021;271(2):235-248.

18. NALLEBALLE K, et al. Spectrum of neuropsychiatric manifestations in COVID-19. Brain, Behavior, and Immunity, 2020; 88: 71-74.

19. OMS. Organização Mundial da Saúde: Doença por coronavírus 2019 (COVID-19) Relatório da situação- 51.2020. Disponível em: https://www.who.int/docs/default-source/coronaviruse/situation-reports/20200311-sitrep-51-covid19.pdf?sfvrsn=1ba62e57_10. Acessado em: 27 de abril de 2020.

20. ROGERS JP, et al. Psychiatric and neuropsychiatric presentations associated with severe coronavirus infections: a systematic review and meta-analysis with comparison to the COVID-19 pandemic. Lancet Psychiatry, 2021; 7(7):611627

21. SZCZESNIAK D, et al. The SARS-CoV-2 and mental health: From biological mechanisms to social consequences. Progress in Neuropsychopharmacology \& Biological Psychiatry, 2021; 104:110046.

22. SULTANA S, ANANTHAPUR V. COVID-19 and its impact on neurological manifestations and mental health: the present scenario. Neurological Sciences, 2020;41(11):3015-3020.

23. TAQUET M, et. al. 6-month neurological and psychiatric outcomes in 236379 survivors of COVID-19: a retrospective cohort study using electronic health records. Lancet Psychiatry, 2021;8(5):416-427.

24. TARSITANI L, et al. Post-traumatic Stress Disorder Among COVID-19 Survivors at 3-Month Follow-up After Hospital Discharge. The Journal of General Internal Medicine, 2021;29:1-6.

25. TESINI BL, Coronavírus e Síndromes respiratórias agudas (COVID-19, MERS e SARS), 2020. Disponível em: https://www.msdmanuals.com/pt/casa/infecções/v\%C3\%ADrus-respiratórios/coronav\%C3\%ADrus-es\%C3\%ADndromes-respiratórias-agudas-covid-19-mers-e-sars. Acessado em 29 de abril de 2020.

26. VARATHARAJ A, et al. Neurological and neuropsychiatric complications of COVID-19 in 153 patients: a UK-wide surveillance study. Lancet Psychiatry, 2020; 7: 875-882.

27. VARGA Z, et al. Endothelial cell infection and endotheliitis in COVID-19. The Lancet, 2020;395:1417-1418.

28. YANG X, et al. Clinical course and outcomes of critically ill patients with SARS-CoV-2 pneumonia in Wuhan, China: a single-centered, retrospective, observational study. The Lancet Respiratory Medicine, 2020; 8: 475-81.

29. ZHAO S, et al. Anesthetic management of patients with suspected or confrmed 2019 novel coronavirus infection during emergency procedures. Journal of Cardiothoracic and Vascular Anesthesia, 2020; 34(5):1125-1131. 